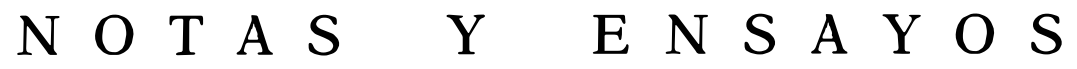

\section{FRANCISCO MOLINER Y NICOLAS \\ (1851-1915) \\ Y EL INICIO DE LA LUCHA \\ ANTITUBERCULOSA EN ESPAÑA}

\section{Jorge Molero Mesa}

Durante los últimos años ha venido observándose un notable interés en los medios académicos hacia la historia social de la tuberculosis (1). La tuberculosis fue durante toda la primera mitad del siglo $\mathrm{XX}$ la enfermedad de mayor prevalencia en los países industrializados. Junto a su alta frecuencia, es de subrayar la selectividad de su acción sobre el brazo productivo de la sociedad. El 80 por 100 de los casos recaía en obreros con edades comprendidas entre los quince y los treinta y cinco años, evidenciando la clara desigualdad existente entre las distintas clases sociales ante la enfermedad y la muerte.

Los diversos estudios que los médicos higienistas españoles realizaron a comienzos de este siglo nos presentan una enfermedad directamente relacionada con las condiciones de vida, la alimentación, el trabajo y la vivienda, fundamentalmente, por lo que su origen, dependiente directamente de la propia organización de la comunidad, no era desconocido ni para las clases dominantes ni para el proletariado como hemos señalado en otro lugar (2). 
Desde esta perspectiva, las diversas administraciones públicas y la propia iniciativa privada organizaron diferentes campañas profilácticas dirigidas a su erradicación. La lucha contra la tuberculosis surgió de una forma definitiva a comienzos del siglo XX en casi todos los países occidentales. En este trabajo estudiaremos los inicios de la campaña antituberculosa en el estado español, en concreto los planteamientos y la experiencia particular llevada a cabo en la Valencia de entresiglos por Francisco Moliner y Nicolás.

\section{DOS PRECEDENTES FRUSTRADOS: ANTONIO ESPINA Y CAPÓ} (MADRID 1889) Y LUIS COMENGE Y FERRER (BARCELONA, 1892)

El primer pronunciamiento colectivo acerca de la prevención de la tuberculosis, sobre la base de su contagiosidad, se realizó en el Congrès pour l'Etude de la Tuberculose chez l'Homme et chez les Animaux celebrado en París del 25 al 31 de julio de 1888 (3). En la conclusión inicial se recogió que la tuberculosis debía inscribirse en los Códigos sanitarios de todos los países entre las llamadas «enfermedades evitables contagiosas", exigiendo además medidas profilácticas especiales. En la segunda se pedía la inmediata hospitalización de los enfermos bacilíferos o infectantes (4).

Dos meses después esta enfermedad fue motivo de atención en el Congreso de Ciencias Médicas de Barcelona (9-15 septiembre de 1888) donde, dentro de un total de nueve, la cuarta ponencia oficial estaba dedicada a discutir la Contagiosidad y profilaxis de la tuberculosis. En esta sesión aunque ningún congresista negaba la existencia del germen, se formaron dos grupos discrepantes en torno al grado de contagiosidad de éste. Para Suñer y Capdevila (mayor) (1826-1898) (5), el contagio estaba probado desde que se inoculó el bacilo de Koch a un moribundo, por lo que la profilaxis de la tuberculosis se debería de realizar mediante dos indicaciones fundamentales: el aislamiento del enfermo y la destrucción de las causas del mal. Junto a Suñer y Capdevila defendían, entre otros, esta tesis Antonio Espina y Capó (1850-1930), Rafael Rodríguez Méndez (1845-1919) e Iranzo (6). Sin embargo, Mariani minimizó el papel del contagio ejemplificándolo en la poca frecuencia con que los médicos padecían la tuberculosis, así como Robert afirmó que, de creer en la alta contagiosidad no hubiera hablado en público «... ante el temor de despertar en el auditorio un sentimiento antihumanitario de horror a los tísicos...». Se dio incluso el caso del Dr. Ezquerdo 
que ignoraba antes de esta sesión que la tuberculosis fuera contagiosa (7).

Esta decisiones, tomadas en las reuniones científicas de la época, inspiraron los fines de las distintas campañas antituberculosas que fueron surgiendo en cada país según sus peculiaridades. Las primeras campañas, por tanto, irían encaminadas a difundir esta nueva doctrina, primero en los círculos académicos y asistenciales y, posteriormente, entre los políticos y el público en general.

En España el primer intento de organizar la lucha antituberculosa se realizó muy tempranamente a manos de Antonio Espina y Capó (8). Presente en el Congreso de París (9) y en el de Barcelona, convocó una reunión en Madrid en diciembre de 1889 con el propósito de fundar una Asociación contra la tuberculosis (10). En la exposición de motivos afirmaba que no sólo los médicos debían de luchar contra los estragos de esta enfermedad sino que

«... incumbe también a todas las representaciones del saber y a todos los que, amantes de su país, estiman como el mejor medio de acrecentar el poder y la riqueza de éste, la conservación y aumento de la densidad de población.»

El objeto de la asociación no podía ser otro que la profilaxis de la tisis ya que «... una vez desarrollada, una vez en potencia en el individuo, poco o nada hemos de conseguir». Proponía un plan de trabajo basado en los siguientes puntos: estudio de la densidad de población en relación con la capacidad de aire, sobre todo en las viviendas de los pobres; saneamiento de las ciudades; creación de arboledas y avenidas; leyes acerca del trabajo excesivo y en la infancia; aumento de coeficiente de alimentación: supresión del hacinamien to en casas, hospitales, cuarteles, cárceles, etc.; exclusión del servicio militar a los mozos con perímetro torácico menor a la talla media, entre otras medidas.

Además subrayó la necesidad de intervenir en los matrimonios, en el sentido de evitar su celebración entre los tuberculosos declarados, ya que «... la tuberculosis no es una enfermedad que pueda dejarse su desarrollo ligado al capricho del legislador o a la inspiración amorosa del individuo». Y por fin la necesidad de destruir todo producto exudado por el tuberculoso y su hospitalización independiente.

En esta misma sesión se constituyó la Junta Central de la Asociación, en la que figuraban nombres tan conocidos como Amalio Gimeno, José de Letamendi, Federico Rubio, Manuel Tolosa Latour, Angel 
Pulido, Alberto Aguilera y su inspirador Antonio Espina, entre otros (11).

A pesar de contar con tan influyentes prohombres esta asociación no prosperó y sólo logró la posterior inscripción de cinco socios (12), quizá por la falta de ambiente contagionista en España respecto a otros países europeos donde estas ideas estaban en boga (13). Así, ante el anuncio de Petit, en el Congreso francés de la tuberculosis al que hemos aludido antes, de crear la Obra de los candidatos a la tuberculosis, instituciones especiales donde serían cuidados los hijos de los tuberculosos pobres apartados de estos, Bassols y Prim la comparó con la "idea ya vieja» del barco para tuberculosos al que todo el mundo llamaría buque de la muerte y donde nadie querría entrar, negando que el aislamiento del enfermo fuese una necesidad social, ya que el contagio no era un factor de primer orden, y tampoco una necesidad individual, pues no estaba demostrado que la hospitalización favoreciera la curación de la enfermedad (14).

En efecto, la idea del contagio, aunque arraigada entre el vulgo, no se encontraba muy extendida entre los facultativos. Por ello, las primeras tareas de los defensores de la contagiosidad de la tisis consistieron en convencer a sus colegas «no confesos», así como a las autoridades. En este punto jugó un papel preponderante la Gaceta Médica Catalana, revista dirigida por Rafael Rodríguez Méndez, quien en 1897 elogiaba al Dr. Moliner por no tener «... reparo alguno en afirmar y en publicar a todos los vientos que la tuberculosis es contagiosa.... (15).

Desde esta óptica contagionista, otro intento de iniciar una campaña antituberculosa fue el protagonizado, en la ciudad de Barcelona, por Luis Comenge y Ferrer (1854-1916). Este, en su calidad de miembro del Cuerpo Médico Municipal, era el responsable de la sección de Higiene Especial y Desinfecciones del Ayuntamiento y, en 1892, el encargado de realizar la estadística demográfica y sanitaria de la ciudad, siguiendo la labor pionera de José Nin y Pullés (16).

En 1892, a través de las páginas de la Gaceta Médica Catalana, hizo un llamamiento a los poderes públicos para que tomaran medidas urgentes de profilaxia contra la tuberculosis (17). Esta premura la justificaba, por una parte, la gran mortalidad que producía la tuberculosis, responsable de provocar una verdadera «sangría suelta» además de que, a pesar de no ser epidémica, había producido más muertes en veinte años que las cuatro epidemias de cólera que en ese mismo período se produjeron en la Ciudad Condal. Por otro lado, la perpetuación del contagio que se mantenía activo por la existencia de casi seiscientas vi- 
viendas contaminadas por enfermedades infecciosas en la ciudad, ya que el vecindario oponía resistencia a las prácticas de desinfección.

Antes estos problemas, Comenge recordó las medidas tomadas en España contra la tisis en los siglos XVII y XVIII, todas concordantes en la obligatoriedad de la declaración de la enfermedad y en la quema o desinfección de ropas y alhajas de los fallecidos por tisis. Solicitó que las autoridades locales acordaran medidas efectivas, y para asesorarles, propuso la creación de una Junta de la que deberían formar parte los catedráticos de Higiene, Patología General, Clínica Médica y Clínica Quirúrgica y el presidente de la Real Academia de Medicina de Barcelona.

Basándose en los edictos y bandos emitidos en los siglos antes citados, Comenge enumeró una serie de medidas «... a título de particular opinión» entre las que destacaba la necesidad de conocer todos los casos de tuberculosis (enfermedad y óbito) para poner en práctica los medios de desinfección (que serían obligatorios), tanto de los enseres como de las viviendas de los tuberculosos. La encargada de realizar estas operaciones sería la Sección de Higiene Urbana del Laboratorio Microbiológico del Ayuntamiento de Barcelona que como hemos visto anteriormente, estaba a su cargo (18).

Junto a estas medidas adjuntó la "prohibición a los tuberculosos de asistir a las escuelas, talleres, fábricas, etc.».

Parte de las normas que propuso Comenge fueron puestas en marcha, un año después, por el Laboratorio Microbiológico Municipal de San Sebastián, cuyo Ayuntamiento había declarado la obligatoriedad de las desinfecciones. Comenge añadió:

«Aquí, en Barcelona, por desgracia, estamos lejos de conseguir este ideal; cuando el año pasado propusimos nosotros (...) la conveniencia de las desinfecciones obligatorias y la prohibición de la venta de ropas en casos de tisis, faltó poco para que nos excomulgaran; se habló de la tiranía de la higiene y se dijo que no era factible lo que proponíamos, cuando todo ello se practicó en siglos anteriores» (19).

El Ayuntamiento de Barcelona terminó tomando medidas similares ese mismo año (20). En una misma disposición en la que se regulaba la asistencia médica gratuita, se notificaba la obligatoriedad por parte de los médicos de declarar a la Alcaldía «... los casos de enfermedad infecciosa que observe... [y] cuantos antecedentes juzgue necesario 
para... combatir el contagio», advirtiendo que a los contraventores de esta norma se les exigirían responsabilidades. La quinta disposición hacía referencia a la desinfección de las viviendas y enseres de enfermos y difuntos de padecimientos infectivos, encomendando esta labor al «... personal de Higiene urbana» (21). También se prohibió comerciar con ropas contaminadas, así como lavarlas en fuentes públicas. Con motivo de no causar resistencia en el vecindario, los servicios, además de ser gratuitos, se harían con «rapidez, sigilo y sin molestias». Por último, el Ayuntamiento se comprometía a indemnizar a los propietarios por los objetos destruidos «... por conveniencia de profilaxis». Estas disposiciones influyeron posteriormente en las iniciativas estatales de normalizar la lucha antituberculosa en España.

La labor iniciada por Comenge tuvo su continuidad con la aparición de la primera revista de tuberculosis que se publicaba en España y una de las primeras revistas científicas en editarse en catalán (22), Contra la Tisi, y cuyo subtítulo rezaba: Nota mensual endressada á popularisar los coneixements contra la tuberculosis. Salió a la luz su primer número en abril de 1903 (23). En efecto, su fundador, Agustí Bassols i Prim, regular colaborador de la Gaceta Médica Catalana, aclaraba que su objetivo era promover el conocimiento entre el pueblo de los movimientos antituberculosos que se estaban realizando en el resto de los países con la formación de distintas asociaciones contra esta enfermedad (24). Esta revista se suspendió temporalmente en enero de 1906 (25) para volver a salir, en una segunda serie, desde junio de 1910 a mayo de 1917, publicada en colaboración con Claudio Bassols (26). El estímulo para su reaparición pudo ser el trabajar en pro de la realización, meses después, de lo que sería la culminación del movimiento catalán antituberculoso, la organización del I Congreso Español Internacional de la Tuberculosis, segundo que se hacía en España y cuya responsabilidad máxima recayó en Rafael Rodríguez Méndez.

\section{CAMPAÑAS DE FRANCISCO MOLINER Y NICOLÁS}

Francisco Moliner y Nicolás (1851-1915), fue el primero que construyó un sanatorio destinado a los tuberculosos pobres en 1899, como después veremos. Su vida profesional siempre estuvo ligada a la Facultad de Medicina de Valencia, primero como ayudante del Museo Anatómico, en 1878, y después como profesor clínico de la misma. En 1883 consiguió la Cátedra de Patología Médica de la Universidad de 
Zaragoza, pero logró, ese mismo año, permutarla por la de Fisiología en la Universidad de Valencia. En 1887 ocupó (parece ser que por encargo) la Cátedra de Patología y Clínica médica de esta misma Universidad, de la que fue Rector en dos ocasiones (1893-1895 y 1897-enero de 1898).

En 1894, en el discurso inaugural de la Real Academia de Medicina y Cirugía de Valencia, Moliner notaba «la falta de amparo» en la que se encontraban las clases sociales desheredadas, sobre las cuales concurrían el mayor número de causas tuberculizantes:

«Van a los talleres y a las fábricas los obreros a ganarse el pan con el trabajo y encuentran allí el aire rumiado y saturado de gérmenes que los envenena; comen poco y mal, se deterioran pronto por los quebrantos del trabajo y las malas reparaciones, pierde a poco su organismo resistencias, y enferma...» (27).

Como contrapartida propuso el confinamiento de los tuberculosos pobres en Granjas-sanatorios donde «... la dirección facultativa, el reglamento, la disciplina y los agentes terapéuticos [aire, sol, reposo, alimentación reparadora]» devolverían la salud a los obreros.

Reconociendo que el principal obstáculo para la realización de este fin era la escasez de dinero, pedía la colaboración del Estado, del mu: nicipio, de la provincia y de «... la caridad de todos por suscripción pública», resaltando el aspecto «útil», además del filantrópico, de que «... un tísico recluido, es un foco menos de infección» (28).

La petición de dinero al Estado sería una constante en los planteamientos de Moliner. Ya en 1896 denunció la cantidad irrisoria que se dedicaba a Sanidad, 480.000 pesetas, inferior a la de años anteriores y de las cuales 350.000 se dedicaban a pagar el sueldo del personal (29). En efecto, los presupuestos del ramo desde 1882 hasta 1888 fueron superiores al millón de pesetas. Esta disminución se ha achacado al alejamiento del peligro de cólera asiático epidémico (30).

Moliner advirtió a los poderes públicos del riesgo que los tísicos pobres, abandonados por la sociedad pudiente, significaban para el orden establecido:

«He aquí el formidable y gravísimo peligro que la tuberculosis vaya ensanchando su círculo de muerte, que la muerte aumente el dolor y la miseria, y que las clases proletarias, apercibidas de que son terciadas por la terrible plaga, pidan con cerebros delirantes por la fiebre 
y pechos henchidos de dolor por la muerte de seres queridos, no lujos, no esplendores... sino en nombre de su derecho a la vida, de su derecho a la salud, al amparo, el auxilio que ahora la sociedad pudiente deja de darles...» (31).

Los supuestos de Moliner exigían que la sociedad prestara socorro a los tuberculosos pobres mediante la construcción de sanatorios populares, comenzando por lograr por parte del Estado la financiación suficiente para construir un Sanatorio Nacional en Porta-Coeli (Valencia) que sirviera de modelo y guía a los centros que debían crearse después en toda España.

Al mismo tiempo, como después veremos, Moliner comenzó las gestiones para organizar una asociación particular que tuviese como fin el combatir la tuberculosis como plaga social.

\subsection{Primera campaña: el "céntimo diario»}

Las esperanzas de Moliner estaban cifradas en conseguir el apoyo de todas las clases sociales, pero fue en la obrera donde encontró mayor eco. En Valencia logró que 14.000 obreros se suscribiesen «a céntimo diario" a su proyecto de financiación del sanatorio lo cual, unido a las colectas que organizaban los estudiantes, hizo que comenzaran las obras del sanatorio en 1898. Poco después, esta labor de propaganda consiguió que se emitiera por parte del Gobierno un Real Decreto por el que se proponía estudiar la viabilidad de convertir al sanatorio de Porta-Coeli en Nacional, y que se le considerase bajo la protección de la Reina y de su hijo Alfonso (32).

Posteriormente, por Real Orden de 3 de marzo de 1899, se reconoció el Sanatorio de Porta-Coeli como obra de "Beneficencia particular" y se nombró una Junta de Patronos para regir dicha institución (33). José Juan Dominé (1871-1931) (34), delegado de la misma, fue el encargado de presentar el sanatorio en la Conferencia Internacional antituberculosa de 1899 celebrada en Berlín (35).

Como continuación de su labor propagandística, Moliner ensanchó sus horizontes, organizando conferencias fuera de Valencia para recabar el apoyo a su campaña. En el mismo año, tras dar una charla en el Centro de Sociedades Obreras de Madrid en la que resaltó cómo la tuberculosis diezmaba siete veces más a la clase proletaria que a las 
clases acomodadas, consiguió el apoyo de los presidentes de las sociedades que formaban el Círculo Obrero. Estos,

\begin{abstract}
«... asumiendo la confianza de más de 13.000 obreros asociados, persuadidos de los beneficios que ha de reportar al Proletariado la creación en España de Sanatorios populares..., unen su voto al de sus compañeros de Valencia... y apoyan las gestiones que hace usted [Moliner] cerca de los Poderes públicos, encaminadas a conseguir que el Estado, con las necesarias subvenciones, transforme el Sanatorio para tísicos pobres que ha fundado en Porta-Coeli, en Sanatorio Nacional»(36).
\end{abstract}

Por su parte, el Partido Socialista, a través de su órgano de expresión, también se adscribió al proyecto de Moliner, con lo que no hacían más que seguir «... el camino que ya nos trazaron otros partidos socialistas, que apoyan en sus países la idea de fundar y sostener por el Estado -único que puede hacerlo- sanatorios nacionales para tuberculosos" (37).

De igual manera también logró Moliner el apoyo de los obreros de Bilbao y Alcoy (38). El total de la campaña del "céntimo diario» llegó a alcanzar una cifra global de 856 talleres inscritos con un total de 140.000 obreros (39).

Hay que señalar que muy pronto, tres semanas más tarde, El Socialista matizó el apoyo dado por los obreros a Moliner. Después de anunciar su regreso a Valencia con el mensaje de los obreros madrileños, el periódico aclaraba que éstos estimaban la obra del sanatorio como de justicia y «de alta conveniencia social» pero sin dar su adhesión completamente ya que, el sanatorio

«no lo quieren ni lo piden a título gracioso; no lo solicitan de la benevolencia ni de la caridad de nadie; no suplican, lo reclaman, lo exigen, como parte de lo mucho que les es debido... Los obreros piden el Sanatorio como cosa a que tienen perfecto e indiscutible derecho; y lo piden con perentoriedad y el imperio de quien reclama lo suyo" (40).

Tras estas puntualizaciones, afirmaba el periódico que seguirían en la campaña para lograr que los céntimos de los obreros madrileños pasaran a engrosar los 14.000 diarios de los valencianos. 


\subsection{La Liga Nacional contra la tuberculosis y de socorro a los tísicos pobres}

La idea de crear una asociación contra la tuberculosis surgió a raíz de una carta aparecida, en 1899, en la recién inaugurada Revista valenciana de ciencias médicas, firmada con el seudónimo de «Dr. $Z$ ». Dicha misiva, dirigida a Moliner, tras apoyar las iniciativas en pro del sanatorio de Porta-Coeli, señalaba la conveniencia de dar un primer paso creando una liga contra la tuberculosis, con pretensiones de convertirse posteriormente en nacional. Esta asociación representaría «... el esfuerzo del instinto nacional de conservación, algo así como un movimiento reparador de la fuerza medicatriz de la colectividad». Finalmente resumía de esta forma sus objetivos:

«sería la liga, en suma, asociación fecunda que tendría como primera parte de su objetivo mantener en todo su vigor la más escrupulosa defensa higiénica y mejorar físicamente al individuo, la familia y la raza, para negar al germen la entrada a el organismo, para combatir lo que solía llamarse en otros tiempos la predisposición» (41).

Un segundo ideal sería buscar el alivio y la curación de los tuberculosos y que, al mismo tiempo, serviría para esterilizar sus productos contaminados, para lo cual proponía la creación de sanatorios.

No tardó Moliner en contestar y, tras agradecer el apoyo que se le brindaba desde esa revista y «quedar a las órdenes del Dr. Z» para la creación de la liga, expresaba su sorpresa al encontrar esta carta, pues venía recién llegado de fuera de Valencia donde había consultado la creación de una asociación de estas características con personalidades médicas de Madrid y Barcelona, que le habían dado su apoyo y el encargo de que trazara en un proyecto las líneas generales de sus posibles estatutos (42).

Después de este intercambio de cartas, Moliner convocó a través de la prensa diaria, a «todas las clases sociales» a una primera reunión para crear la Liga Nacional contra la tuberculosis y de socorro a los tísicos pobres (43). Reunión que, como reconoció su secretario general Ruiz García, no fue muy numerosa. Esta tuvo lugar el 9 de junio de 1899 en el Instituto Médico Valenciano, institución que sería su principal valedora (44). Se aprobaron los estatutos que habían sido redactados por Moliner y se eligió una junta interina que debería dar forma legal a esta nueva institución. Como resultado, fue elegido presidente 
Vicente Peset, que también lo era del Instituto Médico Valenciano. Como vicepresidentes se eligieron a Faustino Barberá, que puso su revista al servicio de la Liga, y a Segismundo Ipa, capellán del regimiento de Vizcaya (45).

Tal como se desprende de su nombre, la Liga tenía por objeto «... combatir la plaga social de la tuberculosis y socorrer socialmente al tísico pobre», para lo cual esta sociedad debía fomentar el estudio científico de dicha enfermedad en todos sus aspectos: clínico, social, experimental y bacteriológico; para después plantear a los poderes públicos el resultado de estas investigaciones de forma que se convirtiesen en leyes de salud pública.

Asimismo, debía favorecer y procurar la instalación de sanatorios populares en «... número proporcionado a la grande extensión de la tisis entre las clases indigentes». Además tendría que recaudar fondos para el mantenimiento de los enfermos en los sanatorios y el sostén de sus familias.

En cuanto a su organización, la Liga estaba abierta a cualquier persona «sean o no médicos» que aceptara sus objetivos y pagara la cuota correspondiente de 25 pesetas para los socios fundadores y de 50 o más para los socios de honor.

Las pretensiones de convertirse en una asociación de carácter «nacional» estaba implícita en su proyecto de estatutos. Se aspiraba a crear juntas o comités en todas las capitales de provincia además de aquellas poblaciones que reunieran un número suficiente de socios. Su internacionalismo quedaba expuesto en la voluntad de inscribirse en el Comité internacional antituberculoso que había sido propuesto por Schrötter (1837-1908) en el Congreso francés de la tuberculosis celebrado en París en 1898 (46).

Además, se proponía la creación de una junta de señoras regidas por los mismos estatutos pero con autonomía en su tesorería y reglamento.

Ahora bien, esta obra debía ser el resultado «... de una función armónica entre la sociedad y el Estado, entre las iniciativas particulares y los poderes públicos». De esta forma, Moliner esperaba que el Estado se hiciese cargo de la mayor parte de la financiación de la campaña.

El 30 de noviembre de 1899, el gobernador civil de Valencia aprobó el reglamento y en la siguiente sesión que se convocó quedó elegida la Junta directiva en la que se reafirmaba en sus puestos a Vicente Peset, Barberá y Segismundo Ipa, eligiéndose como secretario general a Ramiro Ruiz (47). Hay que destacar la presencia en esta Junta directiva, 
como vicesecretario, de José Chabás Bordehore (1877-1963), colaborador de Moliner en el sanatorio de Porta-Coeli y posterior fundador de una de las primeras revistas españolas especializada en tuberculosis, la Revista de Higiene y Tuberculosis (48).

En enero de 1900 se creaban las comisiones de Propaganda, presidida por Sanchís Bergón, de Legislación, por Vicente Dualde y la de Socorros por Manuel Oller (49).

$\mathrm{Al}$ año de constituirse la Liga, su secretario general reconocía que todos los esfuerzos de esta asociación habían estado centrados en tareas de organización interna, lo que unido a la falta de fondos había reducido al mínimo su incidencia social, pese a que, en ese año, ya contaba con 82 socios (50).

\subsection{El sanatorio de Porta-Coeli}

La cura higiénico-dietética en «establecimientos cerrados» destinada exclusivamente a los tísicos, fue definitivamente instaurada, en 1859, por Hermann Brehmer (1826-1889) en Görbersdorf (51). Posteriormente, en 1876, su discípulo Peter Dettweiler (1837-1904) abrió un segundo sanatorio en Falkenstein, que sería imitado por el resto de los países europeos (52).

En España, Zabala, médico del Balneario de Cestona en 1864, recomendaba a los tísicos pasar cinco o seis meses en Málaga y dos o tres en los Pirineos, donde la estación de Panticosa era muy celebre para curar la tisis pulmonar (53). Precisamente el primer sanatorio para ricos de nuestro país fue abierto en el balneario de Busot (Alicante) en 1897. Era propiedad del Marqués del Bosch y estaba bajo la dirección médica de Francisco Valenzuela (54). La estancia en estos centros, así como el resto de los tratamientos clásicos, sólo podía ser seguido por enfermos pudientes dado su elevado coste (55).

Por su parte el problema de la financiación de los sanatorios populares hacía inviable este tipo de instituciones, sólo costeables en Alemania debido a la existencia del seguro obligatorio encargado de mantenerlos. No obstante Moliner, con la ayuda de sus campañas y apoyado por las subscripciones y los estudiantes de medicina (56), consiguió abrir el sanatorio el 15 de julio de 1899 , con capacidad para 14 enfermos. Era el primero que se inauguraba en España dedicado a albergar adultos tuberculosos sin recursos (Tolosa Latour había fundado en Chipiona un sanatorio marítimo para niños en 1892 ), y todo su personal 
subalterno había sido elegido entre repatriados procedentes de las últimas posesiones coloniales (57).

El régimen de vida del sanatorio de Porta-Coeli aparecía recogido en la prensa de la siguiente forma:

«Los enfermos se levantan a las 6 de la mañana, se les da una ducha de lluvia y toman desayuno, consistente en bizcochos y jerez; a las siete se les da café con leche y pan con manteca, y salen al monte, permaneciendo en la pinada hasta las doce. A las diez se les sirven en el mismo monte pasteles de carne o empanadas, y a las doce y media comen con arreglo al siguiente menú: puré, cocido, carne, pescado, fruta, dulce y café. De una a tres es la siesta, y a esta hora vuelven al paseo por la montaña, acompañados del practicante y dos criados, que les llevan catres de campaña para descansar; a las cinco meriendan jamón, salchichón o leche, según prescripción facultativa y el gusto del enfermo, y a las siete regresan al sanatorio donde tienen hora y media de asueto. A las ocho y media se les da la cena, compuesta de tortilla, carne, pescado y postres, y a la diez se retiran a dormir» (58).

En mayo de 1901 el sanatorio había atendido a 320 enfermos que, sumando sus ganancias ponderales, habían engordado alrededor de mil kilos. Moliner, según sus gráficas palabras, afirmaba que el sanatorio repartía «... carne viva y sana» (59). Esta sobrealimentación fue criticada por Vicente Peset, quien la consideraba exagerada y en contra de las costumbres españolas, además de augurar al sanatorio un fracaso económico de seguir derrochando el dinero en ofrecer seis comidas diarias. Por el contrario el método de «refinado sibaritismo» utilizado para combatir la anorexia y que consistía en utilizar «audiciones fonográficas y musicales» durante las comidas le pareció una idea excelente (60).

La atención a los enfermos estaba a cargo de un practicante médico y otro farmacéutico, dos enfermeros, dos ordenanzas, dos limpiadoras y el personal de cocina que, en 1901, se elevaba a 10 miembros (61). En cuanto a los médicos estaba dirigido por Moliner y tenía como jefe clínico a José Chabás (62).

El sanatorio contaba con una revista de igual nombre y de la que tenemos noticia a través de otras publicaciones periódicas en las que se anunciaba como «periódico semanal, propagandista del sanatorio de pobres y defensor de las reformas sociales en favor del proletariado" (63).

Al final de 1899, el Gobierno, mediante Real Decreto, declaraba PortaCoeli de «utilidad pública» (64) y se admitía una proposición de Ley para convertir en nacional el sanatorio (65). 
Para este proyecto de Ley, el Gobierno había consultado previamente a varios organismos de Valencia, entre los que se encontraban la Diputación, el Ayuntamiento y la Sociedad Económica de Amigos del País. Finalmente quedó pendiente del informe del director general de Sanidad, Francisco Cortejarena, que visitó Porta-Coeli en abril de 1900 (66). En su informe afirmaba estar a favor de los sanatorios pero «no uno en determinada provincia, sino en todas aquellas que reúnan las condiciones necesarias», apuntando que los recursos para construir este tipo de centros debían ser aportados por los gobiernos y la caridad de los particulares, aunque reconocía que:

«...el sanatorio de Porta-Coeli representa en España el comienzo de la nueva era en el tratamiento de la tuberculosis y un eficaz auxilio y protección debidos a las clases trabajadoras y enfermos pobres, que por esta condición son los más dignos de que el país y la sociedad en general ayuden y cooperen a los fines que la ciencia se propone para que sus beneficios se distribuyan con la equidad posible en lo humano entre todas las clases sociales» (67).

\subsection{Segunda campaña: ley protectora a los tísicos pobres}

Moliner, tras el rechazo del Gobierno a subvencionar y reconocer a Porta-Coeli como sanatorio nacional, comenzaría de nuevo otra campaña, pero con un enfoque más amplio: ya no pedía para su sanatorio, sino que pretendía que se legislara a favor de la creación de sanatorios populares en todo el Estado español. Su proyecto incluía los siguientes enunciados:

"Artículo 1. ${ }^{\circ}$ Se declara obligación del Estado la creación y fomento de los Sanatorios populares en número suficiente para albergar y dar salud a todos los tísicos pobres de España.

Artículo 2. ${ }^{\circ}$ Se consignarán en cada presupuesto dos millones de pesetas cuando menos para atender a la realización inmediata de lo que prescribe el artículo anterior.»

Finalmente, en el artículo $3 .^{\circ}$ recogía la creación de una Junta Técnica "votada por delegados médicos», a su vez designados por los Colegios Médicos y las Facultades de medicina, la cual ajustaría los fines de esta ley con los "preceptos y sucesivos adelantos de la ciencia» (68).

La campaña consistió en una recogida de adhesiones entre particu- 
lares, colectivos e instituciones. Si se trataba de corporaciones, tales como Ayuntamientos, Universidades, fábricas o colegios, pedía que se le enviase a Valencia y antes del primero de noviembre (fecha de apertura de las Cortes) un acta que certificara el acuerdo corporativo de apoyar dicha ley. A los particulares pedía una instancia en los mismos términos (69).

Una vez en su poder las actas, Moliner pretendía presentarlas a las Cortes reclamando el proyecto de Ley para esa legislatura. En dicho momento, los peticionarios deberían dirigir telegramas al Presidente del Congreso y a la Mayordomía de Palacio, expresando el apoyo a dicho proyecto.

El soporte propagandístico de Moliner estaba basado en la edición de folletos de redacción diversa, según sus destinatarios (Ayuntamientos, estudiantes, etc.). En ellos, tras un discurso apologético de sus campañas anteriores y enumerar las justificaciones por las cuales era necesaria esta ley, daba instrucciones para proceder a la adhesión, incluso cómo redactar los telegramas de apoyo. Esta campaña tuvo también eco entre los medios periodísticos, tanto en la prensa diaria como en la profesional (70).

Por su parte, El Socialista daba la noticia de la «nueva campaña de agitación» de Moliner y de su petición de apoyo a los obreros y aconsejaba a éstos que se la dieran pero sin olvidar «el principal objetivo de su existencia", ya que Porta-Coeli "es simplemente un paliativo", afirmando que: «... la tuberculosis no desaparecerá, ni acaso disminuirá, sino cuando un reparto más justo de las riquezas evite la degeneración por miseria y también la degeneración por exceso de goces»; pero finalmente reconocía que «trabajar por las mejoras en el trabajo y aun por lograr la revolución» no impedía que se auxiliara la creación de sanatorios para que se supiera que «... ansiamos ver convertido en deber del Estado lo que hoy es producto de la iniciativa y del apoyo individual» (71).

A pesar de los «catorce tomos de 10.000 adhesiones a la Ley» y de los «millares de telegramas que llovieron sobre el Congreso», tampoco fue atendida esta campaña de Moliner (72).

Gracias a su popularidad, en 1901 Moliner fue elegido diputado con una campaña encaminada a potenciar el sanatorio de Porta-Coeli y defender, directamente en las Cortes, la «Ley general protectora de todos los tísicos pobres de España» (73). Para defender su candidatura, denominada "humanitaria y social», editó un panfleto en el que se recogían algunas de las adhesiones que hasta la fecha había recibido y un 
mensaje de los enfermos del sanatorio pidiendo el voto para Moliner como única forma de evitar el cierre del sanatorio. Según sus propias palabras era:

\footnotetext{
«... la primera vez que va a las urnas una nueva doctrina de paz y de amor, de ciencia y de progreso predicada con hechos y deseada con tristísimos deseos de dolor y de angustia por los 50.000 tísicos pobres que hoy mueren en España» (74).
}

Su éxito electoral no se vio recompensado en las Cortes donde sus propuestas fueron rechazadas. El Partido Liberal que le nombró Rector de la Universidad valenciana en dos ocasiones, no olvidaba el escándalo que produjo en Valencia el que organizara una corrida de toros bajo su rectorado y que le costó la dimisión de este cargo. En esta legislatura no sólo no se aprobó ninguno de sus proyectos sino que, por el contrario, se vio sometido a un expediente de incapacidad por locura, que aunque no prosperó, pretendía separarle de su cátedra (75).

El nuevo Gobierno, por su parte, acababa de nombrar, en marzo de 1901, a Angel Pulido Director General de Sanidad, quien propuso los primeros decretos contra la tuberculosis. Una extensa Circular (ROC de 4 de octubre de 1901), dirigida a todos los gobernadores civiles, reconocía la importancia de la enfermedad tuberculosa y la imposibilidad del Estado de atenderla. Este documento hacía un llamamiento a todas las personas «... que se interesen por el vigor de su raza y por la conservación de su propia vida...» para que organizaran la lucha antituberculosa y emprendieran una gran labor propagandística en torno a los modos de transmisión y la forma de evitar la enfermedad. Esto desmostraba la falta de voluntad del gobierno de iniciar por cuenta propia la lucha antituberculosa, e ignoraba no sólo la labor emprendida por Moliner y sus secuaces en Valencia, sino todos los pronunciamientos que se produjeron antes de esta fecha y de los que hemos dado cuenta.

Al mismo tiempo, la DGS encargaba a Verdes Montenegro, que fue el fundador del primer dispensario en España en 1901, una "ampliación» de la circular para extender entre la sociedad el convencimiento del mal que significaba la tuberculosis, así como los medios de luchar contra ella. Este estudio fue publicado dentro de la serie monográfica que editaba la DGS. En el prólogo Pulido afirmaba que la doctrina que Verdes defendía era «... la corriente, la del día y hay que aceptarla por el momento según aparece» (76). Verdes, que junto con Espina y Capó 
había sido enviado a Londres en 1900 para que representaran oficialmente al Estado en el Congreso Internacional de la Tuberculosis, fue influido por la comunicación que en dicho congreso presentó Calmette sobre el funcionamiento de los dispensarios. Valoró especialmente sus ventajas frente al sanatorio por «... no exigir los gastos considerables de instalación... para los cuales no parece estar hecha todavía la opinión en España, no obstante la suma de esfuerzos prodigiosa que ha dedicado a tan humanitaria propaganda mi amigo y paisano el Dr. Moliner» (77).

El mismo Verdes se pronunció sobre la imposibilidad de satisfacer las necesidades de cobertura sanatorial a los tuberculosos. Calculaba que, dados los 120.000 enfermos existentes en España, harían falta 1.200 sanatorios de 100 plazas cada uno para aislarlos a todos; lo que consideraba un «... absurdo acerca del cual no es necesario insistir» (78) añadiendo que «la idea de un sanatorio nacional es por completo irrealizable. Apenas si hay necesidad de razonar esta afirmación después de declarado el número de tuberculosos existente», ya que no se podría internar a todos ni sería tampoco conveniente. De todas formas no rechazaba la idea de crear algunos sanatorios para casos incipientes, lo que reduciría el número de candidatos a ingresar en estos centros. Ahora bien

«... la fórmula práctica de hacerlo se obtendría fácilmente modificando un tanto la proposición sometida al Congreso por el infatigable propagandista Dr. Moliner. Encomiéndese a la iniciativa de Diputaciones, Ayuntamientos o Sociedades Benéficas la construcción y sostenimiento de estos centros.»

A continuación pedía la cooperación de asociaciones obreras, grandes empresas y del público en general «... y contribuya con algo el Estado" (79).

En febrero de 1902, después de no aprobarse una subvención anual de 50.000 pesetas pedidas en 1901 (80), el Consejo de Patronos decidió el cierre del sanatorio valenciano debido al «... estado angustioso de sus fondos", despidiendo a los treinta enfermos que para entonces albergaba y aclarando que no era por falta de voluntad de sus bienechores sino porque «... el estado de cultura social, todavía no presta para tales avances» (81). En estas fechas, la Liga antituberculosa ya había dejado de funcionar, no explícitamente, sino por inanición. En 1906, cuando se estaba constituyendo una nueva agrupación de acuerdo con 
la Asociación Antituberculosa Española (AAE) creada en Madrid, se recordó a la antigua Liga para que su «mísera vida de poco más de un año» sirviera de advertencia para la que entonces se creaba (82).

De esta forma iban quedando descartadas las proposiciones de Moliner a quien, no obstante, el Gobierno liberal le admitió, en noviembre de 1902, un nuevo proyecto de Ley, en el que se recogía la obligación del Estado de crear sanatorios populares y fomentar y proteger los dispensarios, esto último novedad en su planteamiento. Según el proyecto se deberían librar 500.000 pesetas anuales, apartando de dicha cantidad 200.000 pesetas como subvención para el sanatorio de Porta-Coeli, que pasaría a ser propiedad del Estado, teniendo en cuenta lo legislado en este sentido, y también «... el plebiscito general elevado a las Cortes en su favor por todas las clases sociales». El proyecto finalmente obligaba a la DGS a organizar un plan de defensa social contra la tuberculosis (83).

Este proyecto de Ley se aprobó en las Cortes "por unanimidad», según Moliner, en diciembre de 1902, pero no llegaría al Senado ya que una crisis política con su correspondiente "cambio de turno" hizo entrar en el Gobierno, el 6 de diciembre, a los conservadores, los que aplazarían sine die la aprobación definitiva de la Ley. En la siguiente legislatura de 1903, Moliner volvió a presentar su candidatura «humanitaria y social» pero esta vez no resultó elegido (84).

El fracaso de las campañas no desanimó a Moliner, quien no desaprovechaba ninguna oportunidad para reiterar sus peticiones, pero cada vez más solitario. En 1903 se había creado en Madrid la AAE con un proyecto de campaña a nivel nacional que contó con el beneplácito del Estado desde el primer momento y que fue, en definitiva, la única institución favorecida por el Gobierno conservador, quien decidió crear, en 1906, la Comisión Permanente de Lucha Contra la Tuberculosis con los miembros de dicha Asociación (85). Moliner colaboró con ellos a nivel organizativo al formar parte de la nueva Liga Antituberculosa Valenciana, creada en 1906, dependiente de la AAE, y que sería la encargada, a partir de entonces, de dirigir la lucha antituberculosa en Valencia por los derroteros de la oficialidad (86). Ya en mayo de 1905, Moliner había intervenido en el mitín organizado por la AAE en el Teatro Real de Madrid. En dicho acto, en el que también intervinieron Santiago Ramón y Cajal y Antonio Espina y Capó, resumió sus diversas actuaciones para conseguir que se aprobase el proyecto de ley de sanatorios populares (87).

Asimismo, consiguió que el Rey visitase Porta-Coeli ese mismo 
año, y que prometiese consignar 25 millones de pesetas en los presupuestos de ese año para la Ley de Sanatorios, hecho que no ocurrió, según Moliner, por la muerte de Villaverde, presidente del Consejo de Ministros. En 1906 el Rey, con motivo de su boda, prometió a Moliner que el primer decreto que firmara sería el de autorizar al Gobierno la presentación del proyecto de Ley, pero esta promesa tampoco se llevó a la práctica (88).

En 1908 sacó a los estudiantes de la Universidad de Valencia a la calle, «... en nombre de la salud de la Patria», para reivindicar que el Gobierno librara un préstamo de 100 millones para Educación y Sanidad, por lo que fue detenido y encarcelado durante un mes (89). Acusado de desórdenes públicos y de coacción e instigación a los alumnos, fue separado de su cátedra en dicha Universidad mediante Real Orden de 28 de julio de ese año (90).

Estos sucesos desembocaron en la presentación por parte de la minoría liberal-democrática del Senado, en junio de 1909, de una proposición de Ley para la emisión de un empréstito para Sanidad y Educación de 100 millones de pesetas. Dicha propuesta fue hecha por el general José López Domínguez, con las firmas de Andrés Mellado, Amalio Gimeno, Bernabé Dávila, Marqués de Santa María y López Muñoz. Sus trámites se congelaron al cerrarse las Cortes como consecuencia de la «Semana Trágica» de Barcelona.

Este empréstito lo justificaba Moliner en la regeneración del proletariado a través de la mejora de sus condiciones higiénicas y culturales:

\footnotetext{
«Ellos se mueren, y ya lo saben, en la proporción de tres por uno; ellos viven, y ya lo saben también, la mitad precisamente de lo que las otras clases sociales viven... ellos son, y también lo saben, esclavos de su ignorancia y carne de presidio por su analfabetismo; ellos saben que el problema de su vida y de su cultura es un problema de dinero... ¿Qué hace la patria que no les devuelve la vida y la sangre que por ella pierden cuando es preciso? ¿Qué hacen esos gobiernos que se llaman previsores y que no previenen el cataclismo que resultará el día en que ellos, que son los más, cansados de sufrir y de pedir, se tomarán la justicia por sus manos?» (91).
}

Los planteamientos de Moliner no habían cambiado. El entendía todas las reformas que pedía como la revolución "de en medio", única que, junto con la «de arriba» (la que le correspondería hacer a los oli- 
garcas), podría frenar "... los pavorosos desastres de sangre y fuego con que la anárquica revolución de abajo amenaza» (92). En el aspecto sanitario, Moliner dio un paso más para la consecución de estos fines al pedir, en 1909, la creación de un Ministerio de Sanidad independiente. Este organismo establecería un "régimen nuevo» de armonía y paz social al atender las enfermedades de los desheredados en la misma medida en que eran atendidas las de los ricos. Su alcance sería tal que no tendría el rango de un simple Ministerio sino que se convertiría en el «quinto Poder del Estado, y se llamaría Poder Sanitario, o el Poder de la Vida y la Salud» (93).

Moliner quemó su último cartucho en el Tercer Congreso Nacional de Tuberculosis, celebrado en 1912 en San Sebastián, en el que aún se creía portaestandarte de los ideales de los obreros valencianos. En su discurso achacó sus fracasos a la fatalidad de sucesos fortuitos producidos por la inestabilidad política del país, de la siguiente forma:

«Sin la crisis de marzo de 1899, catorce años ya que habría en PortaCoeli el mejor Sanatario popular de Europa. Sin la crisis política de diciembre de 1902, diez años ya que tendría España una Ley de Sanatorios populares con cinco millones de pesetas de subvención anual. Sin la muerte de Villaverde, en 1905, la tendríamos con 25 millones. Sin la bomba de la calle Mayor, la tendríamos de cincuenta. Sin la semana sangrienta de Barcelona, desde 1909, que sería Ley del Reino ese crédito de cien millones de pesetas que ahora piden los obreros de Valencia para Cultura y Sanidad» (94).

Despues de pronunciar su discurso, propuso a la asamblea que aprobase la siguiente conclusión, que no había aparecido entre las leídas por el presidente de la Sección correspondiente:

«El Congreso, habida consideración de las razones expuestas en su mensaje por las Sociedades Obreras de Valencia, aconseja al Gobierno la conveniencia, en nombre de la ciencia, de transformar cuanto antes en Ley la proposición de los cien millones para Escuelas y Sanatorios que presentó al Senado el general López Domínguez, en 2 de julio de 1909» (95).

La respuesta del presidente de la Sección de Higiene y Acción social, el abogado José Elosegui, justificaba que los presidentes de las secciones al reunirse y proponer los temas habían tenido que amoldarse a «... las tristes realidades de la vida nacional» redactando la conclusión de la siguiente manera: 
"El Congreso ve con simpatía lo que demanda el mensaje de obreros de Valencia y aconseja al Gobierno procure atenderla.»

Moliner, en un tenso discurso, respondió protestando por la «presión burocrática» que pudiera tener el Congreso, logrando finalmente que la asamblea en pleno votase su propuesta, que fue rechazada por mayoría (96).

\section{NOTAS}

(1) Este fenómeno, iniciado con la reimpresión, en 1987, del clásico libro de René y Jean DuBos: The white plague: tuberculosis, man and society. New Brunswick-London, Rutger University Press, (editado originalmente en 1952 en Boston), se ha concretado muy recientemente con la aparición de monografías que han presentado esta problemática en el contexto de los diversos ámbitos nacionales. Entre ellas destacamos las obras de Bryder, L. (1988): Below the Magic Montain. A Social History of Tuberculosis in Twentieth-Century Britain. Oxford, Clarendon Press, 288 pp. y SмIтн, F. B. (1988): The retreat of tuberculosis, 1850-1950, London-New York-Sydney, Croom Helm, 271 pp., referentes a Gran Bretaña; Teller, M. E. (1988): The Tuberculosis Movement. A Public Health Campaign in the Progressive Era. New York, Greenwood Press, 182 pp., para América del Norte; DesserTine, D.; FAURE, O. (1988):Combattre la tuberculose, 1900-1940. Lyon, Presses Universitaires de Lyon, 244 pp., para Francia; SeEliger, W. (1988): Die "Volksheilstätten-Bewegung" in Deutschland um 1900: zur Ideengeschichte der Sanattoriumstherapie für Tuberkulöse, München, Profil, y REINICKE, P. (1988): Tuberkulosefür sorge. Der Kampf gegen eine Geissel der Menschheit. Dargestellt am Beispiel Berlins 1895-1945, Weinheim, Deutscher Studien Verlag, para Alemania. Para la experiencia española destacamos nuestros trabajos Molero MESA, J. (1987): Estudios medicosociales sobre la tuberculosis en la España de la Restauración. Madrid, Publicaciones del Minis terio de Sanidad y Consumo (Colección textos clásicos españoles de la Salud Pública, n. $\left.{ }^{\circ} 25\right), 377$ pp. y Molero Mesa, J. (1989 $\mathrm{a}$ ): Historia Social de la Tuberculosis en España (1889-1936). Granada, Universidad de Granada, 8 microfichas, 534 pp.

(2) Cf. Molero MeSA, J. (1989b): «La tuberculosis como enfermedad social en los estudios epidemiológicos españoles anteriores a la Guerra Civil». Dynamis, 9, 185-224.

(3) "CONGRESO para el estudio de la tuberculosis humana y animal» (1888), Gaceta Médica Catalana, 10, 759-760. Es de destacar la presencia de Jean Antoine Villemin (1827-1892) como vicepresidente del Congreso. La presidencia estaba ocupada por JeanBaptiste Auguste Chauveau (1827-1917).

(4) ESPINA Y CAPO, A. (1921): La Conferencia de París en octubre de 1920 para fundar la Asociación Internacional contra la Tuberculosis. Conferencia dada bajo la presidencia de S. M. la Reina D. ${ }^{a}$ Victoria Eugenia en la RANM. Madrid, Minuesa hijos, 318 pp. (cita de p. 12).

(5) Este médico, autor de un Tratado popular de la tisis que conoció dos ediciones (1872 y 1899), se llamaba Francisco, igual que su hermano, también médico, por lo que en medios académicos se especificaba si era el «mayor» o el «menor» de los hermanos. SANCHEZ MARTINEZ, G. (1985): Francisco Suñer y Capdevila. Una propuesta materialista para 
la segunda mitad del siglo XIX español (Guerra a Dios, a la Tisis y a los Reyes). Madrid, Tesis de Licenciatura, Universidad de Madrid, ms., 510 pp. (cita de p. 18).

(6) Fargas y Roca, M. A. (1906): "Discurso de contestación». En: Oliver Aznar. Esta do actual de la lucha antituberculosa. Barcelona, Imp. F. Badía, pp. 54-70 (cita de p. 64).

(7) «CONGRESO de Ciencias Médicas de Barcelona» (1888), Gaceta Médica Catalana, 12, 161-166.

(8) Médico de la Beneficencia Provincial de Madrid desde 1876, ingresó en 1898 en la Real Academia Nacional de Medicina a través de la cual fue elegido Senador del reino en 1918.

(9) Este Congreso sería reconocido por Espina como el lugar y momento donde se inició la lucha antituberculosa de la mano de Petit y su Obra de los candidatos a la tuberculosis. EsPINA Y CAPO, A. (1912): "Prólogo». En: LUCHA contra la tuberculosis en España (La), Madrid, Ministerio de la Gobernación, pp. VIII-IX.

(10) La noticia de esta reunión fue recogida en: "ASOCIACION contra la tuberculosis» (1890), Gaceta Médica Catalana, 13, 13-14. Posteriormente este manifiesto sería reimpreso y utilizado para reiniciar la campaña en: EsPINA Y CAPO, A. (1903): Asociación contra la tuberculosis. Exposición de motivos hecha por el Dr.... para justificar su constitución. Madrid, Imp. Nicolás Moya, 8 pp. También sería reimpreso como apéndice en EsPINA Y CAPO (1921), pp. 81-91.

(11) El resto de la Junta estaba compuesto por: Jesús Alcolea, Mariano Araus, Ricardo Egea, Eusebio Castelo, José Fontana, José Francos Rodríguez, Enrique Isla, José La Presilla, Santiago Lavilla, Manuel Martínez Aguiar, Andrés Mellado, Antonio Mendoza, Eusebio Molina, Matías Nieto y Serrano, José Ortega Munilla, Rafael Ulecia y el marqués de Valdeiglesias. A su vez recibió las adhesiones del rector de la Universidad Central, director de la Revista de Terapéutica y Farmacia y de Rodríguez Méndez director de la Gaceta Médica Catalana. "ASOCIACION contra la tuberculosis» (1890), pp. 13-14.

(12) ESPINA Y CAPO (1921), p. 13. Este autor recuerda que, de haberse llevado a cabo, hubiera sido la primera en crearse en el mundo, ya que el movimiento asociativo lo inició en Viena Von Schrötter en 1890. Posteriormente, en 1892, se crearía la Ligue ou Association Centrale Française contre la Tuberculose y la Pennsylvania Society for the prevention of tuberculosis. COURY, Ch. (1972): Grandeur et déclin d'une maladie. La tuberculose au cours des ages. Suresnes, Lepetit S. A., 264 pp. (cita de p. 184).

(13) Espina y Capó convocó otra reunión en 1903 con idéntica finalidad, pero esta vez con mayor fortuna. El 8 de octubre se creó la Asociación Antituberculosa Española (AAE), grupo utilizado para crear el primer organismo oficial español en la materia: la Comisión Permanente de Lucha contra la Tuberculosis establecida por Real Decreto en 1906.

(14) BASSOls I Prim, A. (1888): "Estudios sobre la tuberculosis a propósito del Congreso que a este objeto se reunió en París en julio del corriente año (conclusión)». Gaceta Médica Catalana, 11, 741-745.

(15) Rodríguez MÉndez, R. (1897): «Revista crítica bibliográfica. Higiene». Gaceta Médica Catalana, 20, 217-218.

(16) Su obra ha sido recientemente estudiada con detalle en RoDRíguEz OCAÑA, E. (1985-86): «La labor estadística de Luis Comenge (1854-1916) en el Instituto de Higiene de Barcelona», Dynamis, 5-6, 279-306.

(17) Comenge y Ferrer, L. (1892a):" "La tuberculosis en Barcelona», Gaceta Médica Catalana, 15, 257-264. Este manifiesto contó con el apoyo de esta revista y de su director, el catedrático Rafael Rodríguez Méndez, Rodriguez MÉnDEZ, R. (1982a): «Nuestra 
adhesión", Gaceta Médica Catalana, 15, 264-265. También fue editada en tirada aparte como soporte de la campaña que se intentaba realizar, COMENGE Y FERRER, L. (1892b): La tuberculosis en Barcelona, Barcelona, Imp. de Amat y Martínez, 16 pp.

(18) Rodríguez MÉNDEZ, R. (1917): Necrología del doctor Luis Comenge y Ferrer... leida en la Real Academia de Medicina y Cirugía de Barcelona. Sesión extraordinaria de 26 de noviembre de 1916. Barcelona, Tip. "La Académica», 128 pp. (cita de p. 40). Esta sección fue creada el 9 de junio de 1891 y entre sus funciones estaban las desinfecciones de enseres y viviendas contaminadas y el análisis de alimentos.

(19) Comenge y Ferrer, L. (1893): «Revista crítica bibliográfica», Gaceta Médica Catalana, 16, 116-117. El responsable del Laboratorio de San Sebastián era Chicote, posteriormente jefe del Laboratorio Municipal de Madrid.

(20) "ASISTENCIA médico-gratuita y desinfección en el caso de padecimientos infecciosos" (1893), Gaceta Médica Catalana, 16, 580-582. Se reproducen en esta revista las Disposiciones Municipales fechadas el 19 de septiembre de 1893 y firmadas por Manuel Henrich (alcalde constitucional) y Ramón Larroca (gobernador civil).

(21) En esta fecha, Barcelona contaba con dos centros de desinfección creados por Comenge, el primero de ellos en 1891, siendo este el primero de España en crearse. Ro. DRIGUEZ MÉNDEZ (1917), p. 56.

(22) Connudella I CAPDEVILA, J. (1970): «Historia de la pneumología a Catalunya». En: Actas I Congrés Internacional Historia Medicina Catalana, Barcelona, Scientia, vol. 2, pp. 149-154 (cita de p. 150).

(23) «PUBLICACIONES recibidas» (1903), Gaceta Médica Catalana, 26, 224.

(24) Bassols i Prim, A. (1903): «Revista crítica de bibliografía», Gaceta Médica Catalana, 26, 549-550.

(25) «NUESTRA correspondencia con la prensa» (1906): Gaceta Médica Catalana,

(26) "CORRESPONDENCIA con la prensa» (1910), Gaceta Médica Catalana, 35, 472 y Calvet i Camarasa, J. M.; Corbella I Corbella, J. (1981): Diccionari biográfic de metges catalans. 3 vols., Barcelona, Fundació Salvador Vives Casajuana (cita del vol. 1, p. 70).

(27) Moliner y NicolÁs, F. (1894): «De las granjas-sanatorios en el tratamiento de los tísicos pobres». En: DISCURSOS pronunciados en la inauguración de las secciones de la RAMC de Valencia en el año de 1894, Valencia, Imp. Ferrer de Orga, pp. $25-36$ (cita de p. 27).

(28) Ibid., p. 35.

(29) Moliner y Nicolás, F. (1896): Aspecto social de la tuberculosis. Discurso leído el día 24 de enero, en el Ateneo Científico de Valencia con motivo de la apertura del curso 1895-1896, Valencia, Imp. Federico Domenech, 60 pp. (cita de p. 17). Este texto ha sido recientemente reeditado en: Molero MESA (1987), pp. 69-89.

(30) Rico-Avello, C. (1969): Historia de la sanidad española (1900-1925). Madrid, E. Giménez, 420 pp. (cita de pp. 28-29).

(31) Moliner y Nicolás (1896): pp. 36-39 (cita literal de p. 39)

(32) Real Decreto de 21 de febrero de 1899. Moliner y NiCOLÁs, F. $\left(1900_{\mathrm{a}}\right)$ : Ley protectora de los tísicos pobres. Pedida a los Poderes públicos, por humanidad, en nombre de la ciencia. Circular de propaganda a los ayuntamientos, en solicitud de su adhesión, Valencia, Imp. Francisco Vives Mora, 16 pp. (cita de pp. 7 y 15-16).

(33) Esta junta estaba presidida por el arzobispo de la Diócesis, ibid., p. 16.

(34) Médico y posteriormente economista. En 1905 codirigió, junto con Chabás, la Revista de Higiene y Tuberculosis (en esa época La Salud Pública); fue elegido diputado 
y senador del Partido Liberal. Presidente de la compañía naviera "Transmediterránea», del Monopolio de Petróleos y fundador del Banco de Crédito Local.

(35) MOLINER y NiCOLAS, F. (1899b): Liga Nacional contra la Tuberculosis y de Socorro a los Tísicos Pobres. Proyecto de sus estatutos y reglamento, Valencia, Tip. Domenech, 20 pp. (cita de p. 8).

(36) «SANATORIO de Porta-Coeli (El)» $\left(1899_{\mathrm{a}}\right)$ : El Socialista, 8 diciembre, p. 3. Este documento, fechado a 30 de noviembre, recoge los nombres de todos los presidentes y de sus respectivas sociedades. Está también firmado por Francisco Diego, en representación de la Agrupación Madrileña del Partido Socialista.

(37) Ibidem. Las ideas de Moliner no eran desconocidas para los lectores de El Socialista, ya que en 1897 publicó por entregas el discurso de Moliner «Aspecto social de la tuberculosis» $(2,9,16$ y 23 de abril; 7 y 14 de mayo). En la presentación el semanario justificaba su inclusión, no sólo porque enseñaba y confirmaba mucha de las opiniones de los socialistas sino por «... ser un terrible latigazo a la despiadada e imprevisora sociedad burguesa”.

(38) MOLINER y NiCOLÁS $\left(1900_{\mathrm{a}}\right)$, p. 7.

(39) Chabás, J. (1901): «Excmo Sr. Dr. D. Francisco Moliner Nicolás», Revista MédicoHidrológica Española, 2, 98-105 (cita de p. 104).

(40) «SANATORIO de Porta-Coeli (El)» (1899b): El Socialista, 29 diciembre.

(41) Dr. Z (1899): «Ligas y sanatorios contra la tuberculosis. Carta al Dr. Moliner», Revista Valenciana de Ciencias Médicas, 1, 107-109 (cita de p. 107).

(42) Moliner y Nicolás, F. (1899a): "Carta del Dr. Moliner en contestación a la del Dr. Z», Revista Valenciana de Ciencias Médicas, 1, 155-157. Los médicos que nombra son: Calleja, Calatraveño, Espina y Capó, Mariani, Tolosa Latour y Lozano, todos estos de Madrid; y Robert, Rodríguez Méndez, Cardenal y Batllés de Barcelona. Después aumentaría la lista de personas consultadas: Busto y Compaired, de Madrid; López de Barcelona y finalmente de Valencia: Ferrer y Julve, Peset, Casanova, Barberá (director de la Revista Valenciana de Ciencias Médicas y presumiblemente el autor de la carta firmada como Dr. Z.), Mollá, Lloret y Orellano.

(43) RuIz GaRcia, R. (1900): Liga Española contra la Tuberculosis y de Socorro a los Tísicos Pobres. Agrupación de Valencia. Memoria y discurso leidos en la primera Junta conmemorativa de su fundación el dia 9 de junio de 1900. Valencia, Imp. Antonio López y Cia., 19 pp. (cita de p. 6).

(44) Así lo afirmó Comenge en la felicitación dirigida a la Liga desde Barcelona. CoMENGE Y FERRER, L. (1899): «Liga Española contra la Tuberculosis y de Socorro a los Tísicos Pobres. Reseña», Gaceta Médica Catalana, 22, 676.

(45) Moliner Y NiCOLÁs (1899b): p. 19. El resto de la Junta quedó constituida como sigue: presidentes honorarios: Nicolás Ferrer y Julve, Julio Magraner y Francisco Moliner. Vocales: Sanchís Tomás, Ramiro Ruiz, Gabriel Llabrés, Cubells y Calvo, Ramón Alapont, Bernardino Landete, Mateo Ginés, Juan Busutil, Eduardo Solano, Pedro Lasarte, Miguel Orellano, Manuel Oller Celda, Enrique Alzaga, José Villó, Salvador Pardo, José Martínez Lechón, Federico Almela, Federico Vañó, Abelardo Lloret, Rafael Ridaura, Joaquín Sola y Luis Delgado.

(46) Von Schrötter, como ya hemos visto, fue el creador de la primera liga antituberculosa nacional en Viena en el año 1890 . Su propuesta de realizar una asociación internacional que agrupara a las diferentes nacionales no cuajó hasta 1902, fecha en la que se creó el Bureau Central Internacional para la Lucha contra la Tuberculosis en Berlín. Coury (1972): pp. 184-185. 
(47) RuIz GaRcía (1900): p. 8. El resto de la junta directiva lo componían: tesorero, Miguel Orellano; contador, Vicente Cubells (que no pudo desempeñar el cargo por motivos que desconocemos); vicetesorero, Ramón Alapont; vicecontador, Bernardino Landete; vocales, Vicente Dualde, Nicolás Sanchís, Francisco Villanueva, Manuel Oller, José Sanchís Bergón y Luis Delgado.

(48) Esta revista representó, algunos años después de su fundación, una voz discordante en los planteamientos de la lucha antituberculosa, defendiendo como única medida para terminar con la tuberculosis el descubrimiento de una vacuna eficaz.

(49) Los vocales de estas comisiones eran: Propaganda, Abelardo Lloret, José Juan Dominé, Pedro Lechón, Francisco Villanueva, Gabriel Llabrés, Vicente Guillén, Enrique Alós, Vicente Martí y Ernesto Briz; Legislación, Juan Busutil, Enrique Alzaga, Federico Almela, Eduardo Solano, Buenaventura Guillén, Alberto Oliart, Federico Vañó, Mateo Ginés y Fernando Martínez; Socorros, Roque Chabás, Nicolás Sanchís, Luis Delgado, Francisco Rubio, Salvador Pardo, Pedro Lasarte, José Martínez Lechón, Joaquín Sola y Rafael Ridaura.

(50) RUIz GARcía (1900): pp. 12-14. En este folleto pueden encontrarse los nombres de los 82 socios de la Liga.

(51) KNOPF, S. A. (1900): Les sanatoria. Traitement et prophylaxie de la phtisie pulmonaire. 2. ${ }^{2}$ ed., Paris, Carré et Naud Eds., 495 pp. (cita de p. 10).

(52) Este médico introdujo la típica chaisse longue para la cura de reposo así como la escupidera de bolsillo para sus enfermos. GARRISON, F. H. (1922): Introducción a la Historia de la Medicina. Vol. 2, Madrid, Espasa-Calpe, 633 pp. (cita de p. 380) y Coury (1972) pp. 115 y 140 .

(53) Zabala, J. M. (1865): "Causas de la tisis pulmonar y medios de evitar o disminuir sus estragos». En: ACTAS de las sesiones del Congreso Médico Español celebrado en Madrid, septiembre de 1864. Madrid, Imp. José M. Ducazcal, pp. 635-637.

(54) Valenzuela, F. (1897): Busot (Alicante). Estación médica de invierno y sanatorio permanente para el tratamiento climatológico de las enfermedades del pecho y para la $\mathrm{cu}$ ración de la tuberculosis por el régimen higiénico. Madrid, Imp. Calle de San Isidro, $84 \mathrm{pp}$.

(55) Benito Alcina, catedrático de Higiene de Cádiz, destacó, en 1880, la baja frecuencia con que se encontraba la tisis entre los miembros de la marina inglesa en comparación con los de tierra. Este médico recomedaba los viajes por mar para la curación de la tisis. Alcina, B. (1880): "Los climas marítimos y la navegación en el tratamiento higiénico de la tisis tuberculosa». En: ACTAS de las sesiones del Congreso Regional de Ciencias Médicas celebrado en Cádiz, 10-14 agosto de 1879, Cádiz, F. de P. Jordán, pp. 359-366.

(56) La Junta de patronos del sanatorio montó una tómbola en la feria de Valencia en 1899 con objetos que se habían pedido previamente a ayuntamientos y diputaciones de toda España, en la que también colaboró la Liga antituberculosa. Tenemos noticias de otra tómbola que organizó la Juventud Obrera y la Cruz Roja en la navidad de 1901. Moliner y Nicolás (1900a) p. 8 y Beltrán Báguena, M. (1946): El doctor Moliner. Comentarios sobre la vida de un médico famoso. Barcelona, Ed. Científico-médica, 272 pp. (cita de pp. 221-222).

(57) Dr. C. S. (1899): "El sanatorio de Porta-Coeli", Revista Valenciana de Ciencias Médicas, 1, 222-223.

(58) Peset y CERvera, V. (1899): «Del régimen alimenticio en los sanatorios españoles para tuberculosos pobres», Revista de Medicina y Cirugía Prácticas, 23, 681-691 (cita de p. 689).

(59) Moliner y Nicolás, F. (1901): Candidatura humanitaria y social de Porta-Coeli. 
Valencia, Imp. F. Martínez Andreu, 8 pp. Los gastos invertidos en el sanatorio desde que se creó en 1889 ascendían a $77.158,20$ pesetas en instalación y mobiliario, mientras que en manutención la cifra alcanzaba 176.241,40 pesetas, lo que nos da una idea de los enormes gastos que tuvo que soportar el sanatorio.

(60) Peset y Cervera (1899): pp. 689 y 691.

(61) Dos cocinerós, un repostero, un pinche, un mozo de limpieza, dos sirvientas de cocina, una jefa de comedor y dos camareros. Moliner y NiCOLÁs (1901).

(62) ChABÁS BERgón, E. (1970): «Un médico e historiador valenciano: José Chabás Bordehore». En: Actas del I Congrés Internacional de Historia de la Medicina Catalana. Barcelona, Scientia, vol. 2, pp. 102-115.

(63) «PORTA-COELI, periódico semanal...» (1900), Gaceta Médica Catalana, 23, 744. La ubicación de esta revista estaba en la calle Felix Pizcueta de Valencia y la suscripción para un trimestre costaba 1,25 pesetas. No hemos localizado ningún número de esta revista y todas las noticias que poseemos sobre ella datan de finales de 1900 .

(64) Real Decreto de 6 de diciembre de 1899. "SANATORIO de Porta-Coeli» (1899), Revista Valenciana de Ciencias Médicas, 1, 372-373.

(65) MOLINER y NiCOLÁs $\left(1900_{\mathrm{a}}\right)$, p. 7.

(66) “Porta-Coeli» (1900a), Revista Valenciana de Ciencias Médicas, 2, 127.

(67) Rabena (1900): "El informe del director general de Sanidad acerca del sanatorio de Porta-Coeli ", Revista Valenciana de Ciencias Médicas, 2, 151-153.

(68) MOLINER Y NiCOLÁs $\left(1900_{\mathrm{a}}\right)$, p. 2.

(69) Rodríguez Méndez, R. (1900): «Ley protectora de los tísicos pobres », Gaceta Médica Catalana, 23, 663-664.

(70) Moliner y Nicolás (1900a ) y Moliner y Nicolás, F. $\left(1900_{\mathrm{b}}\right)$ : Ley protectora de los tísicos pobres. Pedida a los Poderes públicos, por humanidad, en nombre de la ciencia. Circular de propaganda a los estudiantes, en solicitud de su adhesión, Valencia, Imp. Francisco Vives Mora, 8 pp.

(71) «PORTA-COELI» $\left(1990_{\mathrm{b}}\right.$ : El Socialista, 9 noviembre.

(72) MOLINER Y Nicolás, F. (1904): Discurso leido en la solemné inauguración de curso de la Universidad Literaria de Valencia (1904-1905). [Tema: mi dimisión]. Valencia, Domenech, 78 pp. (cita de p. 60).

(73) Anteriormente, en 1889, se había presentado a las elecciones con « una original propaganda extrapolítica e idealista " pero quedó relegado al segundo lugar, según Chabás, por un "pucherazo» de los pueblos vecinos. CHABÁs (1901), p. 101.

(74) MOLINER y NiCOlÁs (1901)

(75) Entre las acusaciones que se le imputaban estaba el haber acudido al entierro del torero Fabrilo siendo Rector, haber empleado los fondos destinados a realizar un retrato del rey en hacerse uno propio. También se le acusaba, como profesor, de absentismo y de no suspender a ningún alumno, entre otras cosas. Intervención del conde de Romanones. Diario de Sesiones del Congreso, n. ${ }^{\circ}$ 105, 25 de enero de 1902. Citado por MOLINER y NiCOLÁs (1904), pp. 7-8. Moliner afirmaba que en el Congreso le llamaban «el loco Dios".

(76) Pulido Fernández, A. (1902): Preámbulo. En: Verdes Montenegro, J.: La lucha contra la tuberculosis. Madrid, Dirección General de Sanidad, serie monográfica n. ${ }^{\circ} 1$, pp. 5-8 (cita de p. 7).

(77) VERDES MONTENEGRO, J. (1902): La lucha contra la tuberculosis. Madrid, Dirección General de Sanidad, serie monográfica n. ${ }^{\circ}$ 1, 85 pp. (cita de p. 59). Tanto la Circular de la DGS como el texto de Verdes han sido recientemente reeditados en: Molero MESA (1987), pp. $217-228$ y $229-280$ respectivamente. 
(78) Ibid., p. 47.

(79) Ibid., p. 51

(80) «PARA Porta-Coeli» (1901), Revista Valenciana de Ciencias Médicas., 3, 255.

(81) «SANATORIO de Porta-Coeli (El)» (1902), Revista Valenciana de Ciencias Médicas, 4, 60. El sanatorio tenía deudas por valor de más de 80.000 pesetas a los abastecedores y por varios anticipos pedidos.

(82) «LIGA Antituberculosa de Valencia» (1906), La Salud Pública, 2, 62-63.

(83) «REAL Decreto autorizando al ministro de la Gobernación para que presente a la Cortes el adjunto proyecto de ley sobre creación de Sanatorios populares para tísicos pobres» (1902), Gaceta de Madrid, 21 de noviembre, pp. 633-634. La fecha del Real Decreto es de 4 de noviembre y está firmada por el ministro de la Gobernación, Segismundo Moret.

(84) Beltrán Báguena (1946): p. 234.

(85) Debemos destacar el hecho de que fuese el Partido Conservador, aunque modestamente, el que crease un organismo para luchar socialmente contra la tuberculosis El Partido Liberal, con un programa mucho más progresista de lo que la historiografía le ha concedido en materia de libertades públicas, religión, enseñanza, fue, sin embar go, más «retrasado», como señala MARTINEz CUADRADO, M. (1981): La burguesía conser vadora (1874-1931), 7. ${ }^{a}$ ed., Madrid, Alianza Editorial (Historia de España Alfaguara VI), 613 pp. (cita de p. 415), en lo referente a legislación social y laboral. La Ley de Accidentes de Trabajo (1900), la Ley de Protección a la Infancia (1904), y la definitiva puesta en marcha de la Instrucción General de Sanidad entre otras, fueron realizadas bajo mandato conservador.

(86) RaBena (1906): «Liga antituberculosa en Valencia», Revista Valenciana de Ciencias Médicas, 8, 126-127.

(87) «LUCHA antituberculosa» (1905), Revista Valenciana de Ciencias Médicas, 7, 192-193.

(88) Moliner Y Nicolás, F. (1913): «Mensaje de las Sociedades Obreras de Valencia al Congreso". En: III Congreso español de la tuberculosis. Segundo con carácter internacional celebrado en San Sebastián 9-16 septiembre 1912. San Sebastián, Soc. Esp. Papelería, vol. 1, pp. 159-168 (cita de pp. 163-164).

(89) BeLtrán BAGUena (1946): pp. 237-244.

(90) Cf. Moliner y Nícolás, F. (1911): Pidiendo una revisión en defensa de la verdad y de la justicia. Valencia, Imp. Sucesores de Emilio Pascual, 44 pp. En 1910 Moliner fue juzgado, resultando absuelto por falta de pruebas y en ese mismo año se le reintegró al escalafón de catedráticos.

(91) Moliner y Nicolás, F. (1909 1 ): Sobre el Empréstito extraordinario de cien millones de pesetas para Instrucción y Sanidad. Discurso leido en la solemne sesión de clausura de la Asamblea Reformista de la enseñanza verificada en Valencia el día 23 de junio de 1909. Valencia, Imp. de Manuel Pau, 15 pp. (cita de pp. 7-8).

(92) MOLINER Y NICOLÁS (1904): p. 27.

(93) Los otros cuatro «poderes» eran: el legislativo, el ejecutivo, el judicial y el armónico o moderador. Moliner y Nicolás, F. (1909b): La Sanidad Pública como quinto poder del Estado. Discurso leído en la solemne sesión de clausura de la Asamblea de Subdelegados de Sanidad verificada en Valencia el dia 12 de junio de 1909. Valencia, Imp. de Manuel Pau, 22 pp. (cita de p. 13).

(94) MOLINER Y NICOLÁS (1913): p. 164.

(95) «SESION de conclusiones» (1913). En: III CONGRESO español de la tuberculosis. Segundo con carácter internacional celebrado en San Sebastián 9-16 septiembre 1912. San Sebastián, Soc. Esp. Papelería, vol. 1, pp. 313-340 (cita de p. 325).

(96) Ibid., pp. 325-326 y 330-331. 